\title{
Non-invasive brain stimulation for dystonia: Therapeutic implications
}

\author{
Roberto Erro ${ }^{1,2}$, Michele Tinazzi $i^{3}$, Francesca Morgante ${ }^{4,5}$, Kailash P. Bhatia ${ }^{2}$
}

1. Center for Neurodegenerative diseases (CEMAND), Department of Medicine, Neuroscience section, University of Salerno, Italy

2. Sobell Department of Motor Neuroscience and Movement Disorders, UCL Institute of Neurology, London, United Kingdom

3. Department of Neuroscience, Biomedicine and Movment Science, University of Verona, Verona, Italy

4. Department of Clinical and Experimental Medicine, University of Messina, Messina, Italy

5. Institute of Molecular and Clinical Sciences, St George's University of London, London, United Kingdom

Word count (abstract/text): 113/4239

Figure/Table/References: 0/3/61

Running title: Non-invasive brain stimulation for dystonia

Keywords: transcranial; magnetic stimulation; current stimulation; TMS; tDCS.

Funding: none

Conflict of interest related to the current work: none

Full financial disclosures:

Roberto Erro received consultancies from Zambon and honoraria from TEVA. Michele Tinazzi has nothing to disclose.

Francesca Morgante has received honoraria as a Consultant \& Advisory Boards from Medtronic and Chiesi. She has received honoraria for speaking from UCB Pharma, Medtronic, Lundbeck, Chiesi, Abbvie.

Kailash P. Bhatia received royalties from publication of the Oxford Specialist Handbook Parkinson's Disease and Other Movement Disorders (Oxford University Press, 2008) and of Marsden's Book of Movement Disorders (Oxford 
University Press, 2012). He received funding for travel from GlaxoSmithKline, Orion Corporation, Ipsen, and Merz Pharmaceuticals.

\section{Correspondence to:}

Dr. Roberto Erro

Sobell department of Motor Neuroscience and Movement Disorders, UCL 33 Queen Square, WC1N 3BG, London, UK.

erro.roberto@gmail.com 


\begin{abstract}
Dystonia is characterized by excessive muscle contractions giving rise to abnormal posture and involuntary twisting movements. Although dystonia syndromes are a heterogeneous group of disorders, certain pathophysiological mechanisms have been consistently identified across different forms. These pathophysiologic mechanisms have been subsequently exploited for the development of non-invasive brain stimulation (NIBS) techniques able to modulate neural activity in one or more nodes of the putative network that is altered in dystonia and the therapeutic role of NIBS has been hence suggested. Here we review all studies that applied such techniques as a therapeutic intervention in any forms of dystonia, including the few works performed in children, and discuss emerging concepts and pitfalls of NIBS.
\end{abstract}




\section{Introduction}

Dystonia is a syndrome characterized primarily by excessive muscle contractions giving rise to abnormal posture and involuntary twisting movements [1]. Dystonia can be classified in a number of ways, according to the age-at-onset, distribution, presence of additional signs, and etiology [1]. The current classification relies on two axes: The first defines the clinical features and phenomenology of dystonia in any given patient, whereas the second addresses etiological factors [1]. In most patients, however, definitive etiological conclusions cannot be reached and the dystonia syndrome is hence referred as to idiopathic. Despite dystonia being a widely heterogeneous group of disorders, certain pathophysiological mechanisms have been consistently identified across different dystonia forms [2,3], at least for those considered idiopathic.

Three main abnormalities, which might be influencing each other, have been construed to represent the pathophysiological substrate of dystonia: loss of inhibition at different levels of the central nervous system, maladaptive (excessive) plasticity, and altered sensorimotor integration (for a review see [4]). These pathophysiological mechanisms might explain some clinical phenomena frequently observed in dystonia such as the motor overflow and the presence of sensory symptoms. Such abnormalities have been demonstrated to occur a various levels of the central nervous system (CNS) and dystonia is currently thought to be a network-disorder involving the sensorimotor cortices, the basal ganglia and, possibly, the cerebellum [5].

The current mainstream symptomatic therapy for dystonia is represented by chemodenervation by means of botulinum toxin (BoNT) injections [2]. However, while success rates in patients with cervical dystonia (CD) or blepharospasm (BPS) are reasonably high, in patients with focal hand dystonia (FHD) outcomes are more often disappointing, also due to frequent adverse effects. Moreover, BoNT might not be sufficient when dystonia is distributed over several body regions, as in many children with generalized dystonia. The 
role of deep brain stimulation (DBS) in dystonia is fairly accepted for some dystonic conditions (i.e., DYT1, DYT6, DYT11, tardive dystonia) and is emerging for others, but not all patients are suitable candidates [6]. Therefore, alternative therapeutic approaches are clearly needed.

The putative pathophysiologic mechanisms of dystonia have been exploited for the development of non-invasive brain stimulation (NIBS) techniques. These would be able to induce plastic changes in one or more nodes of the altered network and possibly reverse the aforementioned abnormalities [7]. The concept of neuromodulation holds onto the hope of translating such NIBS techniques into novel therapeutic strategies for dystonia [7]. Two other review articles [8,9] have been previously produced about the therapeutic use of NIBS in dystonia, but both focused on patients with FHD only. Therefore, after providing basic principles of NIBS, we will here review all articles using NIBS techniques for therapeutic purposes in any form of dystonia, including the works performed in children. We will only focus on NIBS techniques (described in details in the next section), hence not covering the applications of DBS for dystonia. The interested readers are therefore referred elsewhere $[6,10]$.

\section{Principles of non-invasive brain stimulation}

Two main techniques are available for human NIBS: transcranial magnetic stimulation (TMS) and transcranial current stimulation (tCS). These neuromodulatory techniques are applied non-invasively over the scalp (figure 1) and hence avoid the possible complications associated with DBS surgery and the side effects of systemic medications [7-9,11,12]. Theoretically, both can be applied over selected cortical regions to modulate the specific corticalsubcortical network that is supposedly linked with a given subset of symptoms. However, there is an established tendency of spread from the target brain area to neighboring areas, which might undermine the topographic selectivity of these techniques. Moreover, both rTMS and tDCS might be uncomfortable for 
patients and can further produce side effects, including the possibility of inducing seizures, which made some authors arguing that the term non-invasive would be inappropriate [13]. Perhaps, the term minimally invasive might be more appropriate.

Beyond these two techniques, there is evidence that (peripheral) transcutaneous electrical nerve stimulation (TENS) can modulate motor cortex excitability [14] and will be therefore discussed here, despite not being classically considered a NIBS technique.

\section{1 rTMS}

Repetitive TMS (rTMS) refers to application of trains of repeated magnetic pulses delivered to the scalp. Passing a brief current through an insulated coil held placed on the scalp surface generates a magnetic field perpendicular to the coil that, in turn, induces a weak current within the underlying cerebral cortex. Commonly used coils have a figure- 8 shape able to stimulate brain cortical areas with a functional spatial resolution of $0.5 \mathrm{~cm}$ to $1 \mathrm{~cm}$ [15]. Using TMS, neuromodulatory effects can be elicited in a number of ways depending mostly on the frequency and/or pattern of stimulation: Low $(<1 \mathrm{~Hz})$ or high $(>5 \mathrm{~Hz})$ frequency rTMS decreases or increases, respectively, cortical excitability. The exact mechanisms by which rTMS modulates the cortical excitability beyond the duration of the stimulation protocol are not clear. It is supposed that these are explained by long-term potentiation (LTP) and long-term depression (LTD) changes at synaptic level [16]. While these changes during the stimulation might be exerted by a direct influence on NMDA-dependent plasticity, late-LTP and late-LTD changes are arguably driven by gene expression regulation and protein synthesis [16].

An alternative method of rTMS is theta burst stimulation (TBS), consisting of short, repeated bursts of TMS pulses at high frequency. In such case, the neuromodulatory effect relies on the pattern of stimulation with continuous TBS 
(cTBS) having inhibitory effects and intermittent TBS (iTBS) facilitatory effects. In actual fact, a single train of TBS induces a mixture of suppressive and facilitatory effects, with facilitation building up faster and suppression being more powerful in the long-term [17]. Since it is assumed that both mechanisms saturate at some level, the results might be explained upon the duration of the stimulation (i.e. as long as inhibition is builds up in the long run) [17]. Accordingly, a short, intermittent protocol as iTBS would favor rapid build-up of facilitation [17]. In contrast, a longer lasting continuous protocol such as cTBS would initially produce facilitation, but this would saturate and inhibitory effects (which build up slower but saturate at higher level) would eventually dominate [17]. This argument is however speculative and the exact mechanisms whereby TBS induces plastic changes are not entirely clear, the effects also depending on the intensity of stimulation [18-20].

It should be noted, however, that there might be a significant amount of interindividual variability in response to such protocols. In fact, some subjects show facilitation after 1-Hz rTMS and others show excitability suppression after 10$\mathrm{Hz}$ rTMS [21]. Some authors suggested a role for genetic polymorphisms in the observed inter-individual variability [22].

\section{2 tCS}

Transcranial current stimulation refers to the application of an eletrical current through a pair of surface electrodes placed onto the scalp. tCS does not induce massive synchronized discharge of action potentials as with TMS techniques (e.g., does not induce activity in resting neuronal networks), but modulates spontaneous neuronal activity $[23,24]$.

Two main tCS techniques have been developed. In the most commonly used technique, the transcranial direct current stimulation (tDCS), a weak direct current delivered through the scalp is able to induce polarity-specific changes of resting membrane potential. That is, cortical excitability is diminished by 
cathodal stimulation which hyperpolarises neurons, while anodal stimulation causes an increase of excitability by depolarizing neurons [23, 25]. The magnitude of the after-effects of tDCS are proportional to the intensity and duration of the applied current. Differently from rTMS, for which the intensity used for any single subjects is based on the individual rest- or active-motor threshold, for tCS (both direct and alternating) there is no such a proxy and the intensity is set at either 1 or $2 \mathrm{~mA}$.

With the transcranial alternating current stimulation (tACS), both electrodes have equivalent effects that are dependent on the oscillating current frequency [24]. Accordingly, $1 \mathrm{~mA}$ tACS with frequency outside the conventional EEG range [e.g., with frequencies of $140 \mathrm{~Hz}$ and in the low $\mathrm{kHz}$ range $(1-5 \mathrm{kHz})$ ] increases excitability similarly to anodal tDCS [26,27]. When tACS is applied with frequency in the EEG range, it is supposed to entrain with or synchronize neuronal networks, and to induce changes in ongoing oscillatory brain activity. The efficacy of the stimulation seems to be also dependent on the power of intrinsic oscillations at baseline [28]. When applied over $\mathrm{M} 1,10-\mathrm{Hz}$ or $15-\mathrm{Hz}$ tACS leads to a pattern of inhibition of cortical excitability [29,30], while $20-\mathrm{Hz}$ tACS increases cortical excitability [31].

\subsection{TENS}

The concept behind TENS stems from the evidence that manipulations of afferent input can induce lasting changes within the primary sensori-motor cortex [32-34]. The central effects of TENS are dependent on the frequency of the stimulation, with low frequency (1-4 Hz) increasing and high frequency ( $>50$ $\mathrm{Hz}$ ) depressing sensori-motor excitability. The suggestion that plastic changes occur centrally is corroborated by the fact that peripheral (M-wave) and spinal (H-wave) excitability remain unchanged after TENS [14]. The exact mechanisms whereby TENS modulate sensory-motor excitability are not clear, 
with some authors speculating it could induce LTP changes at inhibitory synapses [14].

\section{Search strategy}

We searched the Medline database (via PubMed, a service of the National Library of Medicine's National Center for Biotechnology Information; http://www.ncbi.nlm.nih.gov) for anytime publications using the following terms: Term $\mathrm{A}=$ [neuromodulation $\mathrm{OR}$ non-invasive brain stimulation $\mathrm{OR}$ repetitive transcranial magnetic stimulation $\mathrm{OR}$ theta burst stimulation $\mathrm{OR}$ transcranial current stimulation OR electrical stimulation OR rTMS OR tCS OR tDCS OR tACS OR TENS] AND term B = [dystonia]. All types of original articles, including case reports, were included if NIBS protocols were performed for therapeutic purposes or had at least one robust clinical outcome (i.e., we excluded pure pathophysiologic studies). Moreover, studies for which details about the stimulation protocol were not fully provided were excluded. Only articles written in English were included. Review articles were checked to include relevant articles not indexed in the electronic database.

\section{NIBS in children with dystonia}

Only three studies [35-37] from the same group evaluated the therapeutic potential of NIBS in children with dystonia, of which only one had a doubleblinded, sham-controlled design. All three used tDCS. A summary of the studies evaluating NIBS in children with dystonia is provided in table 1.

In 2012 Young et al. performed an open-label study with cathodal tDCS in 10 children with dystonia due to different etiologies (e.g., 2 patients had idiopathic dystonia, whereas in the remaining dystonia was secondary to a variety of causes, table 1) using a single-session cathodal stimulation over the motor cortex contralateral to the most affected side [35]. They failed to demonstrate any 
improvement on the Barry-Albright Dystonia (BAD) scale, but observed a nonsignificant reduction of the motor overflow during an electromyogram tracking task [35]. Hence, they repeated the experiment on 14 children with dystonia using a double-blinded, sham-controlled design with cathodal tDCS [36]. Similarly to the above, there was no significant clinical change as measured by the BAD scale, but they found a significant reduction in the motor overflow, although the effect size was admittedly small [36]. Finally, Bhanpuri et al. attempted to explore whether repeated tDCS sessions could lead to a cumulative effects with meaningful clinical results in 9 patients, again with different etiologies accounting for their dystonia syndromes [37]. Using a double-blinded, sham-controlled design, they assessed over 5 sessions whether cathodal or anodal stimulation over the motor cortex contralateral to the most affected side could be beneficial. They failed to demonstrate any clinical and electromyogram changes [37]. However, as observed in prior studies, there was a great intersubject variability and individual analysis suggested that cathodal stimulation over the motor cortex could be in fact beneficial, whereas anodal tDCS even worsened motor performance in some patients. The major pitfall of all 3 studies is the inclusion of very heterogeneous patients (table 1), which hampers drawing definitive conclusions. Although the authors could not identify a clear pattern of response between "primary" and "secondary" patients, sample sizes were very small to allow such type of comparison. Therefore, larger, double-blinded and sham-controlled studies would be required in homogenous groups of patients. From a practical standpoint, it is to be acknowledged that in all 3 studies a small subset of patients did not tolerate the intensity of the stimulation (initially set between 1 and $2 \mathrm{~mA}$ ), so that it was reduced below $1 \mathrm{~mA}$ and all patients but one could complete the protocol. No major side effects were observed, largely in line with results obtained in other clinical populations of children or adolescent [38]. 


\section{NIBS in adults with dystonia}

\subsection{Focal hand dystonia}

\subsection{1 rTMS}

Eight studies were examined [39-46], of which only one had a double-blinded design. A summary of these studies is provided in table 2. There was a high heterogeneity in terms of design (open-label, single-blinded, with or without sham sessions and/or cross-over) and of patients included. The majority of studies included patients with writer's cramp (WC) with the rationale of applying inhibitory protocols to the cortical motor areas contralateral to the affected side. Siebner et al. found that a single-session of $1 \mathrm{~Hz}$ rTMS over M1 reduced writing pressure and this was somewhat mirrored by a normalization of the cortico-cortical inhibition and a prolongation of the cortical silent period (CSP) [39]. Similar results were obtained by Murase et al. in a single-blinded, sham-controlled study using $0.2 \mathrm{~Hz}$ rTMS over the premotor cortex (PMC), but not M1 or supplementary motor area (SMA) [41]. The therapeutic role of low-

frequency rTMS over PMC has been investigated in different studies. A pilot TMS-PET study on seven FHD patients employed a single session $1 \mathrm{~Hz}$ rTMS for 30 minutes over the dorsal PMC (dPMC) and evaluated perfusion before and after the magnetic stimulation. Although cerebral blood flow was decreased at a larger extent in lateral and medial premotor areas, putamen, and thalamus after inhibitory rTMS of dPMC in dystonic patients, neither handwriting nor global clinical score improved [40]. Conversely, Borich et al. showed a significant improvement of handwriting performance (lasting up to 10 days after the end of the stimulation period) by inhibiting the PMC with 5 consecutive $1 \mathrm{~Hz}$ rTMS sessions [42]. Importantly, such an improvement was not observed after a single session, suggesting cumulative effects with repeated sessions. In contrast to the above, Kimberley et al. failed to show any clinical improvement targeting the PMC with 5 consecutive sessions of $1 \mathrm{~Hz}$ rTMS [45]. However, their 
experimental group was heterogeneous as it included 7 patients with WC and 5 with musicians' dystonia (MD) [45]. The flaw of patient heterogeneity was somewhat investigated in another study from Kimberley et al. [46] which, despite yielding negative results, showed self-rated improvement with large effect sizes (suggesting clinical meaningfulness) in some subjects. The authors therefore advocated the need of identifying baseline predictors to distinguish responders from non-responders [46]. The therapeutic role of PMC in FHD has been further questioned by Huang et al. [44] who adopted a single-blinded, sham-controlled design with 5 consecutive sessions of cTBS over the PMC and failed to show any clinical changes, despite a significant increase in cortical inhibition. Finally, the study by Havrankova et al. was the only one adopting a double-blind design with cross-over [43]. The authors assessed whether 5 consecutive sessions of $1 \mathrm{~Hz}$ rTMS over S1 could be beneficial in 11 patients with WC and they observed an improvement in both subjective and objective measures of handwriting lasting up to 2 weeks after the stimulation period, although no changes were observed for the BFMDS score [43].

\subsection{2 tDCS}

Seven studies were included [47-53] (table 2), all of which but one had a double-blinded design [52]. In two consecutive studies, Buttkus et al. showed that single-sessions of both cathodal and anodal tDCS over M1 contralateral to the affected side are not effective in patients with MD [47,48]. Similar (negative) results were obtained in 12 patients with WC by Benninger et al. [49], who applied cathodal tDCS over M1 for three 20-minute sessions over one week. Conversely, Furuya et al. succeeded in showing a significant improvement in patients with MD targeting M1 bilaterally, but only when the stimulation was performed during motor (re)training and the cathode was placed over the affected hemisphere [50]. This would suggest that a combined use of bi-hemispheric tDCS with bimanual motor retraining is required to elicit 
improvement of rhythmic accuracy of a trained sequence of finger movements. Similar results were obtained by Rossett-Llobet et al. [52], who used repetitive sessions of bi-hemispheric tDCS over the sensorimotor cortices (e.g., 5 consecutive sessions/week for two consecutive weeks) combined with sensorimotor training. They showed that the combination of bi-hemispheric tDCS (with the cathode applied contralateral to the affected side) significantly increase the effectiveness of the rehabilitation programs. Finally, two studies assessed the potential therapeutic role of cerebellar tDCS [51,53] yielding somewhat contradictory results. Indeed, Bradmann argued for an improvement of handwriting kinematics following anodal tDCS of the ipsilateral cerebellum [53]. However, no clinical changes were observed using validated dystonia severity scales. Moreover, there was observed an unexpected decrease of cerebellar-brain inhibition (CBI), leaving the question open of what the mechanisms underlying the kinematic improvement are. Similarly, Sadnicka et al. did not show any clinical improvement or cortical excitability changes in 10 patients with WC after anodal tDCS over the ipsilateral cerebellum [51]. Given that high heterogeneity in terms of cortical excitability responses was observed, the authors claimed that inter-subject variability might undermine cerebellar stimulation as a therapeutic strategy for FHD [51].

\subsubsection{TENS}

TENS applied on forearm flexor muscles was used in a randomized, doubleblind, placebo-controlled crossover study in $10 \mathrm{WC}$ patients [54]. The frequency of stimulation was of $50 \mathrm{~Hz}$ and intensity was below the pain threshold and muscular contraction. Electrical stimuli were administered throughout each 20minute session in 2-second trains (100 stimuli/train) separated by 2 -second pauses. The authors found that 10 sessions in 2 weeks improved some objective 
and subjective aspects of writing, although no change was seen in a disability score [54]. The effect lasted for about 6 weeks [54]. In a subsequent study it was demonstrated that hand writing improvement after one TENS session on the forearm flexor muscles was paralleled by a significant reduction of MEP from the flexor muscles and an increase of the MEP from the extensor muscle in 10 WC patients [55].

\subsection{Craniocervical dystonia}

\subsection{1 rTMS/cTBS}

Three studies using either rTMS or cTB were performed in patients with cervical dystonia (CD) [56-58], whereas only one used rTMS in patients with blepharospasm [59]. An overview of these studies is provided in table 3. Koch and colleagues used repeated session of cTBS targeting the lateral cerebellum bilaterally in a double-blind, sham-controlled fashion over two weeks in a total of $18 \mathrm{CD}$ patients [56]. They found a significant reduction of the at the end of the protocol for the real cTBS but not for sham stimulation [56]. However, the benefit was not maintained at 2- and 4-week follow-up [56]. In addition, they demonstrated a reduction of heterotopic paired associative stimulation excessive facilitation following cTBS, suggesting that modulation of CBI can indeed modify some of the pathophysiological substrates of dystonia and lead to a clinical improvement [56].

Pirio Richardson et al. [57] performed a single-blinded, sham-controlled, low frequency rTMS study on a number of cortical areas to find out the best target to use in CD dystonia. Despite non-significant, the larger improvements on the TWSTRS were observed following rTMS over M1 and dPMC [57]. Zittel and colleagues applied a similar protocol of low frequency rTMS over S1/M1 and 
demonstrated that short-latency afferent inhibition (SAI) could be normalized although this did not reflect any changes in symptoms severity [58].

Finally, Kranz et al. performed a double-blind, sham-controlled study on 12 BPS patients using a single session low frequency rTMS study [58]. The target was set on the Anterior Cingulate Cortex (ACC) in the point of maximal MEP for the orbicularis oculi muscle. They found that all clinical outcomes (both patient- and clinician-rated) improved along with a "normalization" of the blink reflex recovery cycle [59].

\subsection{2 tCS}

Only two case-reports $[60,61]$ have been published thus far, of which one with the use of tDCS and the other one with a mixed design using both tDCS and tACS. In 2011 Angelakis et al. showed that $15 \mathrm{~Hz}$ tACS over C3/C4 (to target the supplemental motor cortex, SMC) was superior to cathodal tDCS over C4 and led to a significant improvement of the clinical picture, with the TWSTRS reducing of about 50\% [60]. In addition, the pain TWSTRS-subscale had a reduction of almost $75 \%$ [60]. It was also shown that the effects persisted one months after the stimulation protocol, suggesting that multiple tACS sessions have cumulative effects [60]. Bradman et al. instead used repeated sessions of anodal tDCS over the cerebellum, bilaterally, and right M1 (e.g. contralateral to the "most affected" side) [61]. They found a reduction of the TWSTRS of about $40 \%$ (the pain TWSTRS-subscale decreased of about 55\%) as well as of other dystonia-specific quality-of-life scales [61]. The clinical results were mirrored by electrophysiological changes in corticomotor excitability [61]. Importantly, the stimulation protocol was performed starting one week after botulinum toxin (BoNT) injections [61], suggesting tDCS has the potential to augment BoNT effects.

\section{Discussion}


Although only few data are available in dystonia yielding somewhat contradictory results (figure 2), the works reviewed here could pave the way for future studies on a larger scale. The aims of the current review were in fact to identify potential issues for the use of NIBS for the treatment of dystonia. As a result, four main questions are to be addressed.

\section{1) Is there any consensus regarding specific NIBS settings to be used?}

The results obtained by the studies reviewed here were often negative and this might undermine the theoretical hypothesis behind the use of these techniques as therapeutic tools. It might well be that the aimed inhibitory effect of NIBS (by means of low-frequency rTMS or anodal tDCS) could not outweigh excessive cortical excitability observed in dystonia, at least with single stimulation sessions. There is preliminary evidence in fact that cumulative effects can be obtained by repeated stimulation over consecutive days $[37,43,60]$. Hence, the results of single-session studies might have been negative simply because of an insufficient number of stimulation sessions [62]. However, it should be also noted that there is no consensus about the setting of these techniques and an incomplete knowledge of the physiological effects of NIBS. As to rTMS, the after-effects are dependent on the frequencies, phases (monophasic/biphasic) [63], intensity (sub-threshold or supra-threshold), on the total number of pulses [64]. Also, the stimulation might be more (or less) focused on the brain target according to the specific type of coil that is used and this might influence the final outcome $[59,65]$. Hence, studies comparing different stimulation settings might be worth as well as works aimed to compare different techniques (i.e. rTMS vs tCS). Finally and quite obviously, accurate positioning of the coil/electrodes on the brain target is critical [43] and different studies adopted different ways for the same target. A consensus in this regard would also enhance comparability across different studies.

2) Is there clarity about the target to be stimulated? 
After the initial work by Siebner et al. showing an improvement in FHD after inhibition of M1 [39], Murase et al. provided evidence that PMC could be a better target and four subsequent studies used the PMC contralateral to the affected side as stimulation target [41]. Subsequent works yielded contradictory results with four studies failing to show significant benefits in objective measures, despite self-rated improvements were reported in some of these studies [40-46]. Interestingly, Borich et al. reported an improvement of handwriting performance, but this was only observed after 5 stimulation sessions [42]. This might imply that PMC could be in fact a promising target but repeated sessions of stimulation would be required for clinically meaningful effects, as proposed above. Finally, S1 has been proved as an efficacious alternative target with the improvement observed in both objective and subjective measures (again after 5 sessions of stimulation) [43]. With regards of tDCS, all studies [47-49,51,53] but two [50,52] failed to show any significant changes either by stimulating M1 or the cerebellum and regardless of the type of stimulation (e.g., chatodal or anodal). Interestingly, the two studies showing a potential benefit of tDCS applied a bi-hemispheric stimulation over the primary sensorimotor cortices with the cathode over the affected hemisphere and in both the stimulation was delivered during the execution of motor retraining [50,52]. It is not entirely clear whether it is the type of montage with bi-hemispheric stimulation or the combination of motor retraining and tDCS to be required to produce the improvement.

The few studies performed in CD also yielded contradictory results. The study by Pirio Richardson [57], similarly to that of Murase in FHD [41], is the only one comparing different stimulation targets in a population of $\mathrm{CD}$. They found that the largest improvement was observed for stimulation of the PMC and M1 [57]. Preliminary positive results (with small size effects) have been demonstrated after cTBS of the cerebellum [56]. As far as tCS is concerned, the two case-report published so far $[60,61]$ have shown promising results and it has 
been claimed that tACS could be superior to tDCS, but there are no studies indicating which should be the best target to stimulate.

Although PMC has been proved as one of the most promising targets for both FHD and CD, it is unclear why this should be the case. PMC is a complex structure that has multiple connections with the SMA, the sensorimotor cortex, the anterior cingulate and the basal ganglia and Tyvaert and colleagues have demonstrated that sensorimotor integration (one of the main pathophysiological abnormalities in dystonia) could be ameliorated by low-frequency rTMS of the PMC [65]. The reasons why stimulating any other nodes of the sensori-motor network has not always produced similar results remain to be determined and further physiological studies are warranted to fully understand the "network effect" of NIBS [13]. Interestingly, there is preliminary evidence that peripheral electrical stimulation would modulate sensorimotor integration and lead to an improvement of dystonia [54,55]. Modulating sensory afferents by means of a protocol that employs the physiological sensory pathway might prove an approach with higher topographic selectivity, as the stimulation can in fact be delivered on the affected body part.

\section{3) Are NIBS suitable only for some dystonia populations?}

As far as children with dystonia are concerned, despite overall negative results, there are some indications that cathodal tDCS over primary sensorimotor cortices can be to some extent beneficial in reducing motor overflow [35-37]. However, it should be noted that: 1) there is a paucity of studies in children with a total population of 33 subjects studied thus far; and 2) there was an impressive heterogeneity in terms of etiology. All three studies performed in children [3537] in fact enrolled subjects with non-progressive CP, secondary (progressive) dystonia and genetic forms such as DYT1, rendering the results hardly interpretable. Moreover, it should be noted that in these studies some children did not tolerate the stimulation at full intensity, so that it was reduced and this, as mentioned above, might have also affected the results. This is a potential 
issue that needs to be considered when evaluating NIBS in children. As for now, there is no robust evidence to consider NIBS for dystonia in children.

As to adults with dystonia, the majority of studies were focused on patients with FHD [39-53]. This is due to the fact that patients with FHD usually respond less to BoNT injections than those with other types of focal dystonia, highlighting the need for novel therapeutic strategies. Moreover, FHD represents an interesting model to explore NIBS given that symptoms are generally confined to one body side. Despite this, there is no clear rationale to argue that only specific dystonia populations would benefit from NIBS, given that the main pathophysiological mechanisms underlying the manifestation of dystonia are shared across different sub-groups [3,4]. However, opposite results have been sometimes demonstrated for different forms of dystonia. For example, low frequency rTMS over S1 has been shown to enhance SAI in CD [58] and to reduce it in WC [66]. Hence, larger studies with homogenous populations are required to estimate the magnitude of benefit in each of these dystonia groups.

4) Are available clinical scales sensitive enough to detect minimal changes?

The final question is whether available clinical scales are in fact sensitive enough to detect minimal significant changes. Quite obviously, different scales were adopted across different studies and this hampers comparisons among them. One strategy to overcome such a hurdle might be considering as primary outcome in pragmatic RTCs patients' willing to continue the treatment, while waiting for more sensitive clinical scale to be developed [67]. On the other hand, clinical assessments should be paralleled by neurophysiological evaluations in order to fully understand the neural circuits that NIBS modulate and possibly identify reliable biomarkers that correlate with dystonic symptom severity.

\section{Conclusions}


In summary, there remain many pitfalls regarding NIBS techniques as therapeutic tools for dystonia and at the current time none can be recommended. Moreover, there is lack of studies assessing whether NIBS can augment the effectiveness of rehabilitation or pharmacological treatments [68]. It is in fact interesting to note that some authors suggested the potential of NIBS to augment the benefit produced by BoNT injections [61], so that NIBS technique could be seen as complementary rather than alternative treatments. At the current stage, NIBS cannot be recommended for use in dystonia populations. However, further studies, possibly exploring novel techniques $[69,70]$, are warranted to see whether NIBS can eventually be implemented in clinical practice.

\section{Table captions}

Table 1. Summary of the studies using NIBS techniques in children with dystonia

CP: Cerebral palsy; TBI: Traumatic brain injury; BAD: Barry-Albright Dystonia Scale; SMA: Supplementary motor area.

Table 2. Summary of the studies using NIBS techniques in focal hand dystonia

WC: writer's cramp; HC: Healthy controls; FHD: Focal hand dystonia; MD: musicians' dystonia; RMT: Rest motor threshold; AMT: Active motor threshold; PMC: premotor cortex; SMA: Supplementary motor area; dPMC: dorsal premotor cortex; SMC: sensorimotor cortices; BMFDS: Burke-Marsden-Fahn Dystonia Scale; WCRS: Writer's cramp rating scale; ADDS: Fahn's Arm Dystonia Disability Scale; CSP: Cortical silent period; BOLD: Blood-oxygenlevel dependent; CBI: Cerebellar-brain inhibition.

Table 3. Summary of the studies using NIBS techniques in craniocervical dystonia 
CD: Cervical dystonia: BPS: Blepharospasm; HC: Healthy controls; RMT: Rest motor threshold; ACC: anterior cingulate cortex; PMC: premotor cortex; SMA: Supplementary motor area; dPMC: dorsal premotor cortex; MEP: Motor evoked potential; TWSTRS: Toronto Western Spasmodic Torticollis Rating

Scale; CDQ-24: Craniocervical Dystonia Questionnaire; CDIP-58: Cervical Dystonia Impact Profile; BMFDS: Burke-Marsden-Fahn Dystonia Scale; PAS: Paired associative stimulation; CBI: Cerebellar-brain inhibition; BRR: Blink reflex recovery cycle.

Figure 1. Summary of NIBS techniques for excitation and inhibition

Figure 2. Graphical summary of the studies using rTMS or tCS

\# Anode over the hemisphere contralateral to the affected side

\section{References}

[1] Albanese A, Bhatia K, Bressman SB, et al. Phenomenology and classification of dystonia: a consensus update. Mov Disord. 2013;28:863-73

[2] Morgante F, Klein C. Dystonia. Continuum (Minneap Minn). 2013;19:122541.

[3] Thompson V, Jinnah HA, Hess E. Convergent mechanisms in etiologicallydiverse dystonias. Expert Opin Ther Targers 2011;15:1387-1403.

[4] Quartarone A, Hallett M. Emerging concepts in the physiological basis of dystonia. Mov Disord. 2013;28:958-67

[5] Niethammer M, Carbon M, Argyelan M, Eidelberg D. Hereditary dystonia as a neurodevelopmental circuit disorder: evidence from neuroimaging. Neurobiol Dis 2011;42:202-209. 
[6] Picillo M, Lozano AM, Kou N, Munhoz RP, Fasano A. Programming Deep Brain Stimulation for Tremor and Dystonia: The Toronto Western Hospital Algorithms. Brain Stimul 2016;9:438-52.

[7] Wagle Shukla A, Vaillancourt DE. Treatment and physiology in Parkinson's disease and dystonia: using transcranial magnetic stimulation to uncover the mechanisms of action. Curr Neurol Neurosci Rep. 2014;14:449.

[8] Cho HJ, Hallett M. Non-Invasive Brain Stimulation for Treatment of Focal Hand Dystonia: Update and Future Direction. J Mov Disord. 2016;9:55-62

[9] Quartarone A, Rizzo V, Terranova C, Milardi D, Bruschetta D, Ghilardi MF, Girlanda P. Sensory abnormalities in focal hand dystonia and non-invasive brain stimulation. Front Hum Neurosci. 2014;8:956

[10] Fox MD, Alterman RL. Brain Stimulation for Torsion Dystonia. JAMA Neurol. 2015;72:713-9.

[11] Tyvaert L, Houdayer E, Devanne H, Monaca C, Cassim F, Derambure P. The effect of repetitive transcranial magnetic stimulation on dystonia: a clinical and pathophysiological approach. Neurophysiol Clin. 2006;36:135-43.

[12] Wu AD, Fregni F, Simon DK, Deblieck C, Pascual-Leone A. Noninvasive brain stimulation for Parkinson's disease and dystonia. Neurotherapeutics. 2008 Apr;5(2):345-61

[13] Davis NJ, van Koningsbruggen MG. "Non-invasive" brain stimulation is not non-invasive. Front Syst Neurosci. 2013 Dec 23;7:76.

[14] Tinazzi M, Zarattini S, Valeriani M, Romito S, Farina S, Moretto G, Smania N, Fiaschi A, Abbruzzese G. Long-lasting modulation of human motor cortex following prolonged transcutaneous electricalnerve stimulation (TENS) of forearm muscles: evidence of reciprocal inhibition and facilitation. Exp Brain Res. 2005;161:457-64

[15] Brasil-Neto JP, Cohen LG, Panizza M, Nilsson J, Roth BJ, Hallett M. Optimal focal transcranial magnetic activation of the human motor cortex: 
effects of coil orientation, shape of the induced current pulse, and stimulus intensity. J Clin Neurophysiol 1992;9:132-136.

[16] Malenka R, Bear M. LTP and LTD: an embarrassment of riches. Neuron 2004;44:5-21.

[17] Huang YZ, Edwards MJ, Rounis E, Bhatia KP, Rothwell JC. Theta burst stimulation of the human motor cortex. Neuron. 2005;45:201-6.

[18] Peinemann A, Reimer B, Loer C, et al. Long-lasting increase in corticospinal excitability after 1800 pulses of subthreshold $5 \mathrm{~Hz}$ repetitive TMS to the primary motor cortex. Clin Neurophysiol 2004;115:1519-1526

[19] Touge T, Gerschlager W, Brown P, Rothwell JC. Are the aftereffects of low-frequency rTMS on motor cortex excitability due to changes in the efficacy of cortical synapses? Clin Neurophysiol 2001;112:2138 -2145.

[20] Fitzgerald PB, Brown TL, Daskalakis ZJ, Chen R, Kulkarni J. Intensitydependent effects of $1 \mathrm{~Hz}$ rTMS on human corticospinal excitability. Clin Neurophysiol 2002;113:1136-1141.

[21] Maeda F, Keenan JP, Tormos JM, Topka H, Pascual-Leone A. Interindividual variability of the modulatory effects of repetitive transcranial magnetic stimulation on cortical excitability. Exp Brain Res 2000;133:425- 430. [22] Kleim JA, Chan S, Pringle E, et al. BDNF val66met polymorphism is associated with modified experience-dependent plasticity in human motor cortex. Nat Neurosci 2006;9:735-737.

[23] Miranda PC. Physics of effects of transcranial brain stimulation. Handb Clin Neurol. 2013;116:353-66

[24] Antal A, Paulus W. Transcranial alternating current stimulation (tACS). Front Hum Neurosci. 2013 Jun 28;7:317

[25] Woods AJ, Antal A, Bikson M, et al. A technical guide to tDCS, and related non-invasive brain stimulation tools. Clin Neurophysiol. 2016;127:103148 
[26] Chaieb L., Antal A., Paulus W. (2011). Transcranial alternating current stimulation in the low $\mathrm{kHz}$ range increases motor cortex excitability. Restor. Neurol. Neurosci 29, 167-175

[27] Moliadze V., Atalay D., Antal A., Paulus W. (2012). Close to threshold transcranial electrical stimulation preferentially activates inhibitory networks before switching to excitation with higher intensities. Brain Stimul. 5, 505-511 [28] Neuling T., Rach S., Wagner S., Wolters C. H., Herrmann C. S. (2012). Good vibrations: oscillatory phase shapes perception. Neuroimage 63, 771-778 [29] Antal A., Boros K., Poreisz C., Chaieb L., Terney D., Paulus W. (2008). Comparatively weak after-effects of transcranial alternating current stimulation (tACS) on cortical excitability in humans. Brain Stimul. 1, 97-105

[30] Zaghi S, de Freitas Rezende L, de Oliveira LM, El-Nazer R, Menning S, Tadini L, Fregni F. Inhibition of motor cortex excitability with $15 \mathrm{~Hz}$ transcranial alternating current stimulation (tACS). Neurosci Lett. 2010 Aug 2;479(3):211-4.

[31] Feurra M., Paulus W., Walsh V., Kanai R. Frequency specific modulation of human somatosensory cortex. Front. Psychol. 2011;2:13

[32] Calford MB, Tweedale R. Immediate and chronic changes in response of somatorysensor cortex in adult flying-fox after digit amputation. Nature.1998; $332: 446-8$

[33] Recanzone GH, Allard TT, Jenkins WM, Merzenich MM. Receptive-field changes induced by peripheral nerve stimula- tion in SI of adult cats. J Neurophysiol. 1990;63:1213-1225

[34] Nudo RJ, Milliken GW, Jenkins WM, Merzenich MM. Use- dependent alterations of movement representations in primary motor cortex of adult squirrel monkeys. J Neurosci. 1996;16:785-807

[35] Young SJ, Bertucco M, Sheehan-Stross R, Sanger TD. Cathodal transcranial direct current stimulation in children with dystonia: a pilot openlabel trial. J Child Neurol. 2013;28(10):1238-44 
[36] Young SJ, Bertucco M, Sanger TD. Cathodal transcranial direct current stimulation in children with dystonia: a sham-controlled study. J Child Neurol. 2014;29(2):232-9

[37] Bhanpuri NH, Bertucco M, Young SJ, Lee AA, Sanger TD. Multiday Transcranial Direct Current Stimulation Causes Clinically Insignificant Changes in Childhood Dystonia: A Pilot Study. J Child Neurol. 2015 Oct;30(12):160415.

[38] Palm U, Segmiller FM, Epple AN, Freisleder FJ, Koutsouleris N, SchulteKörne G, Padberg F. Transcranial direct current stimulation in children and adolescents: a comprehensive review. J Neural Transm (Vienna). 2016; 123:1219-34

[39] Siebner HR, Tormos JM, Ceballos-Baumann AO, Auer C, Catala MD, Conrad B, et al. Low-frequency repetitive transcranial magnetic stimulation of the motor cortex in writer's cramp. Neurology 1999;52:529-537.

[40] Siebner HR, Filipovic SR, Rowe JB, Cordivari C, Gerschlager W, Rothwell JC, Frackowiak RS, Bhatia KP. Patients with focal arm dystonia have increased sensitivity to slow-frequency repetitive TMS of the dorsal premotor cortex. Brain. 2003;126:2710-25

[41] Murase N, Rothwell JC, Kaji R, Urushihara R, Nakamura K, Murayama N, et al. Subthreshold low-frequency repetitive transcranial magnetic stimulation over the premotor cortex modulates writer's cramp. Brain 2005;128(Pt 1):104115

[42] Borich M, Arora S, Kimberley TJ. Lasting effects of repeated rTMS application in focal hand dystonia. Restor Neurol Neurosci 2009;27:55-65 [43] Havrankova P, Jech R, Walker ND, Operto G, Tauchmanova J, Vymazal J, et al. Repetitive TMS of the somatosensory cortex improves writer's cramp and enhances cortical activity. Neuro Endocrinol Lett 2010;31:73-86. 
[44] Huang YZ, Lu CS, Rothwell JC, Lo CC, Chuang WL, Weng YH, et al. Modulation of the disturbed motor network in dystonia by multisession suppression of premotor cortex. PLoS One 2012;7:e47574.

[45] Kimberley TJ, Borich MR, Arora S, Siebner HR. Multiple sessions of lowfrequency repetitive transcranial magnetic stimulation in focal hand dystonia: clinical and physiological effects. Restor Neurol Neurosci 2013;31:533-542. [46] Kimberley TJ, Schmidt RL, Chen M, Dykstra DD, Buetefisch CM. Mixed effectiveness of rTMS and retraining in the treatment of focal hand dystonia. Front Hum Neurosci 2015;9:385.

[47] Buttkus F, Weidenmüller M, Schneider S, Jabusch HC, Nitsche MA, Paulus W, et al. Failure of cathodal direct current stimulation to improve fine motor control in musician's dystonia. Mov Disord 2010;25:389-394.

[48] Buttkus F, Baur V, Jabusch HC, de la Cruz Gomez-Pellin M, Paulus W, Nitsche MA, et al. Single-session tDCS-supported retraining does not improve fine motor control in musician's dystonia. Restor Neurol Neurosci 2011;29:8590.

[49] Benninger DH, Lomarev M, Lopez G, Pal N, Luckenbaugh DA, Hallett M. Transcranial direct current stimulation for the treatment of focal hand dystonia. Mov Disord 2011;26: 1698-1702.

[50] Furuya S, Nitsche MA, Paulus W, Altenmüller E. Surmounting retraining limits in musicians' dystonia by transcranial stimulation. Ann Neurol 2014;75:700-707.

[51] Sadnicka A, Hamada M, Bhatia KP, Rothwell JC, Edwards MJ. Cerebellar stimulation fails to modulate motor cortex plasticity in writing dystonia. Mov Disord 2014;29:1304-1307.

[52] Rosset-Llobet J, Fàbregas-Molas S, Pascual-Leone Á. Effect of transcranial direct current stimulation on neurorehabilitation of task-specific dystonia: a double-blind, randomized clinical trial. Med Probl Perform Art 2015;30:178-184 
[53] Bradnam LV, Graetz LJ, McDonnell MN, Ridding MC. Anodal transcranial direct current stimulation to the cerebellum improves handwriting and cyclic drawing kinematics in focal hand dystonia. Front Hum Neurosci 2015;9:286.

[54] Tinazzi M, Farina S, Bhatia K, Fiaschi A, Moretto G, Bertolasi L, Zarattini S, Smania N. TENS for the treatment of writer's cramp dystonia: a randomized, placebo-controlled study. Neurology. 2005;64:1946-8.

[55] Tinazzi M, Zarattini S, Valeriani M, Stanzani C, Moretto G, Smania N, Fiaschi A, Abbruzzese G. Effects of transcutaneous electrical nerve stimulation on motor cortex excitability in writer's cramp: neurophysiological and clinical correlations. Mov Disord. 2006;21:1908-13

[56] Koch G, Porcacchia P, Ponzo V, Carrillo F, Cáceres-Redondo MT, Brusa L, Desiato MT, Arciprete F, Di Lorenzo F, Pisani A, Caltagirone C, Palomar FJ, Mir P. Effects of two weeks of cerebellar theta burst stimulation in cervical dystonia patients. Brain Stimul. 2014 Jul-Aug;7(4):564-72

[57] Pirio Richardson S, Tinaz S, Chen R. Repetitive transcranial magnetic stimulation in cervical dystonia: effect of site and repetition in a randomized pilot trial. PLoS One. 2015 Apr 29;10(4):e0124937.

[58] Zittel S, Helmich RC, Demiralay C, Münchau A, Bäumer T. Normalization of sensorimotor integration by repetitive transcranial magnetic stimulation in cervical dystonia. J Neurol. 2015;262(8):1883-9.

[59] Kranz G, Shamim EA, Lin PT, Kranz GS, Hallett M. Transcranial magnetic brain stimulation modulates blepharospasm: a randomized controlled study. Neurology. 2010 Oct 19;75(16):1465-71

[60] Angelakis E, Liouta E, Andreadis N, Leonardos A, Ktonas P, Stavrinou LC, Miranda PC, Mekonnen A, Sakas DE. Transcranial alternating current stimulation reduces symptoms in intractable idiopathic cervical dystonia: a case study. Neurosci Lett. 2013 Jan 15;533:39-43. 
[61] Bradnam LV, Frasca J, Kimberley TJ. Direct current stimulation of primary motor cortex and cerebellum and botulinum toxin a injections in a person with cervical dystonia. Brain Stimul. 2014;7:909-11.

[62] Monte-Silva K, Kuo MF, Liebetanz D, Paulus W, Nitsche MA. Shaping the optimal repetition interval for cathodal transcranial direct current stimulation (tDCS). J Neurophysiol 2010;103(4):1735-40.

[63] Hosono Y, Urushihara R, Harada M, Morita N, Murase N, Kunikane Y et al. Comparison of monophasic versus biphasic stimulation in rTMS over premotor cortex: SEP and SPECT studies. Clin Neurophysiol 2008; 119:25382545.

[64] Antonini A, Morgante F. Repetitive transcranial magnetic stimulation in the treatment of dystonia. In: Stacy M, editor. Handbook of dystonia. London: Informa Healthcare; 2012. p. 501-11.

[65] Tyvaert L, Cassim C, Devanne H, et al. Subthreshold low frequency rTMS over the premotor cortex and sensorimotor integration in patients with writer's cramp. Neurology 2006;66:A179

[66] Bäumer T, Demiralay C, Hidding U, Bikmullina R, Helmich RC, Wunderlich S, Rothwell J, Liepert J, Siebner HR, Münchau A. Abnormal plasticity of the sensorimotor cortex to slow repetitive transcranial magnetic stimulation in patients with writer's cramp. Mov Disord. 2007;22:81-90.

[67] Comella CL, Fox SH, Bhatia KP, Perlmutter JS, Jinnah HA, Zurowski M, McDonald WM, Marsh L, Rosen AR, Waliczek T, Wright LJ, Galpern WR, Stebbins GT. Development of the Comprehensive Cervical Dystonia Rating Scale: Methodology. Mov Disord Clin Pract. 2015 Jun;2(2):135-141.

[68] Nitsche MA, Müller-Dahlhaus F, Paulus W, Ziemann U. The pharmacology of neuroplasticity induced by non-invasive brain stimulation: building models for the clinical use of CNS active drugs. J Physiol. 2012;590(19):4641-62. 
[69] Erro R, Rocchi L, Antelmi E, Palladino R, Tinazzi M, Rothwell J, Bhatia KP. High frequency repetitive sensory stimulation improves temporal discrimination in healthy subjects. Clin Neurophysiol. 2016;127(1):817-20 [70] Rocchi L, Erro R, Antelmi E, et al. High frequency somatosensory stimulation increases sensori-motor inhibition and leads to perceptual improvement in healthy subjects. Clin Neurophysiol. 2017. In press 